\title{
Pratiques culturales et diversité des insectes en cultures vivrières à Korhogo (Nord, Côte d'Ivoire)
}

\author{
Mouhamadou KONE ${ }^{1 *}$, Yalamoussa TUO ${ }^{1,}$ Michel Laurince YAPO ${ }^{1}$, \\ Yégnon Adjaratou $\mathrm{SORO}^{2}$, Namory DOSSO ${ }^{1}$ et Bebe KAMBOU ${ }^{1}$ \\ ${ }^{1}$ UFR Sciences Biologiques, Université Peleforo Gon Coulibaly, Korhogo, Côte d'Ivoire, BP 1328 \\ Korhogo, Côte d'Ivoire. \\ ${ }^{2}$ Institut de Gestion Agropastoral, Université Peleforo Gon Coulibaly, Korhogo, Côte d'Ivoire, BP 1328 \\ Korhogo, Côte d'Ivoire. \\ "Auteur correspondant: Email: kmouhamadou2@yahoo.fr; kmouhamadou@upgc.edu.ci; \\ Tel.: + 22505355531 .
}

\section{RESUME}

Pour contribuer dans l'amélioration de la production vivrière dans le département de Korhogo (au nord de la Côte d'Ivoire) par une gestion efficace des ravageurs de cultures, une étude a été réalisée pour déterminer l'abondance et la diversité des insectes. Cette étude avait pour objectif d'évaluer l'impact de deux systèmes culturaux différents (une monoculture et une polyculture) sur la diversité des insectes. Les méthodes d'échantillonnage des insectes utilisant les pièges-fosses et les pièges colorés ont été appliquées. Les résultats obtenus ont montré une abondance nettement élevée d'insectes dans la parcelle de monoculture (4112 individus) par rapport à la polyculture (725 individus). Parmi les groupes d'insectes récoltés, les Hyménoptères, les Orthoptères et les Hémiptères sont clairement concentrés dans la monoculture. Toutefois, l'analyse de la similitude entre les compositions taxonomiques montre une forte ressemblance entre les deux types de cultures. De cette étude, il ressort que la polyculture peut contribuer à limiter la prolifération des ravageurs en ce sens qu'elle est source de perturbations dans la gestion des stimuli chez les insectes ; ce qui limite le maintien de leur peuplement.

(c) 2018 International Formulae Group. All rights reserved.

Mots clés : Diversités, insectes, monoculture, polyculture, ravageurs, cultures vivrières.

\section{Cultural practices and diversity of insect in food crops at Korhogo (North, Côte d'Ivoire)}

\begin{abstract}
We assessed food crops improvement in northern of Cote d'Ivoire (Korhogo) by handling crop pest practices. The aim of this study consists at investigating the impact of two different cultural systems (i.e. monospecific culture and mixed culture) on insect diversity. For this purpose,
\end{abstract}


sampling was implemented by combining pitfall trapping and coloured plate sampling. Results revealed a high proportion of insect in a monospecific culture (4112 individuals) as opposed to mixed culture system (725 individuals). Of insect orders collected, Hymenoptera, Orthoptera and Hemiptera were clearly most abundant in monospecific culture. Findings suggested a relative similitude between both cultural procedures with regard collected insect's taxonomic composition. Overall, mixed culture can contribute at reducing pest increasing because it is source of disturbance for insect stimuli management.

(C) 2018 International Formulae Group. All rights reserved.

Keywords: Diversity, insect, monospecific culture, mixed culture, pests, food crop plantations.

\section{INTRODUCTION}

Les performances de l'économie ivoirienne restent encore dépendantes du secteur primaire notamment de l'agriculture dont la contribution au PIB se situe aux alentours de $25 \%$. La production vivrière a connu une croissance considérable en 2012 avec un bilan qui se situe autour de 10 millions de tonnes (FAO, 2015).

Les cultures vivrières entrent directement dans l'alimentation en assurant la vie des populations humaines et du bétail d'où leur appellation de «produits vivriers ». Représentées par le riz, le maïs, le mil, le sorgho, le fonio, l'igname, la patate douce, le tarot, etc.... Elles sont produites par les populations rurales, majoritairement les femmes et sont destinées à l'autoconsommation, même si une quantité relativement importante est commercialisée (Anader, 2009). Nombreuses sont les raisons qui permettent d'encourager la production vivrière. Tout d'abord, ce sont des aliments de base polyvalents permettant d'assurer la sécurité alimentaire et nutritionnelle ; ensuite, beaucoup parmi ces spéculations sont des cultures à cycle court et elles ont une grande adaptation agro-écologique; enfin, elles constituent des aliments de base bon marché, mais riches d'un point de vue nutritif, qui offrent un apport en protéines, vitamine $\mathrm{C}$, vitamine $\mathrm{A}$, zinc et fer permettant de répondre aux besoins alimentaires des villes.

Comme toutes les spéculations agricoles, les cultures vivrières sont confrontées à des défis liés aux pertes dues aux insectes ravageurs (Mailafiya et al., 2014 ;
Choudourou et al., 2012; Diatte et al., 2016). L'importance des dégâts occasionnés aux cultures et aux denrées stockées par ces organismes, contraint l'agriculteur à recourir à des mesures de protection. Parmi celles-ci, la lutte chimique à l'aide des pesticides de synthèse a longtemps été considérée comme la solution la plus efficace et la plus facile à mettre en œuvre. Cependant, leurs effets secondaires sur l'environnement et la santé ne sont pas compatibles avec une exploitation durable des agroécosystèmes. Des solutions alternatives sont donc recherchées, notamment la prévention des risques considérée aujourd'hui comme un préalable nécessaire. On est ainsi conduit à concevoir une stratégie phytosanitaire nouvelle reposant sur la gestion agro-écologique des populations et des peuplements d'insectes. Cette stratégie remet en cause les pratiques habituelles et nécessite une prise en considération des associations culturales largement répandue sous les tropiques (Hautier et al., 2003).

La polyculture réduit le nombre de certains ravageurs en renforçant l'abondance de leurs ennemis naturels. Selon Bianchi et al. (2006), Chaplin-Kramer et al. (2011), et Chaplin-Kramer et al. (2013), les paysages diversifiés détiennent plus de potentiel pour la conservation de la biodiversité et le maintien de la fonction de contrôle des ravageurs.

$\mathrm{La}$ polyculture entraine une perturbation des stimuli olfactifs. Ainsi, les odeurs de certaines plantes aromatiques ont permis de repousser des ravageurs dans des vergers de chou et de coton. Selon Auger et al 
(2002), les propriétés pesticides des composés volatils des Allium semblent considérables. Ces composés apparaissent comme potentiellement utilisables pour le contrôle des ravageurs tels que les insectes phytophages, les acariens, les nématodes. Au Nigeria, il a été constaté que l'infestation était réduite dans des champs où la tomate était associée avec des céréales, des tubercules ou d'autres légumes (Umeh et al., 2002). Si sous d'autres cieux, cela fait l'objet de plusieurs études, il n'en est pas le cas en Côte d'Ivoire où les associations culturales restent encore un mode

\section{MATÉRIEL ET MÉTHODES}

\section{Sites d'étude}

L'étude a été réalisée dans le département de Korhogo, au nord la Côte d'Ivoire, plus précisément dans le village de Gnalélékaha. Avec une superficie de 12500 $\mathrm{km}^{2}$, le département de Korhogo se trouve entre $5^{\circ} 16$ et $6^{\circ} 16$ de longitudes Ouest et $8^{\circ} 32$ et $10^{\circ} 20$ de latitudes Nord. Le climat est de type soudanien ou tropical subhumide caractérisé par deux types de saisons: une saison sèche qui s'étend de novembre à mai, le mois de janvier étant généralement le plus sec et une saison des pluies qui dure de juin à octobre où les mois de juillet, août et septembre sont les plus pluvieux. La température moyenne annuelle dans le département de Korhogo est de $27^{\circ}$ C. La végétation rencontrée est essentiellement constituée de forêts claires et de savanes. Les principales essences utiles rencontrées sont le Néré (Parkia biglobosa) et le Karité (Vitellaria paradoxa).

Les insectes ont été échantillonnés dans deux types de pratiques culturales (monoculture et polyculture) afin de déterminer leur richesse, leur abondance et leur composition taxonomique. L'échantillonnage a été réalisé d'Avril en juillet 2018.

Les champs de monocultures ( 3 parcelles) échantillonnés étaient situés dans le village de traditionnel de culture pratiqué par des petits paysans sur de petites surfaces.

L'objectif général de cette étude est d'évaluer l'impact de deux systèmes culturaux différents (une monoculture et une polyculture) sur la diversité des insectes en vue d'une optimisation des rendements. Spécifiquement il s'est agi de déterminer la richesse et l'abondance des insectes dans deux systèmes de cultures différents (monoculture et polyculture) et de comparer leur composition taxonomique.

Gnalélékaha qui est à environ deux kilomètres de Korhogo. Ils étaient constitués uniquement de patate douce d'environ deux mois. Les cultivateurs étaient en majorités des femmes et elles utilisaient des dabas pour l'entretien des champs. Certains insecticides (Lambdacyhalotrine $15 \mathrm{~g} / 1+$ Acétamipride20g/1;

Cyperméthrine $100 \mathrm{~g}$ ) et herbicides (2,4- d de sel d'amine) étaient également utilisés dans cette culture (Figure 1).

Les champs de polycultures échantillonnés étaient localisés dans le même lieu que les champs de monocultures. Ce système cultural était composé d'association de maïs et de patate douce d'environ deux mois (Figure 2).

\section{Méthode d'échantillonnage des insectes}

La collecte des insectes a été effectuée à l'aide de deux méthodes standardisées : celle des pièges-fosses et celle des pièges colorés (assiettes jaunes). Ces méthodes de piégeages occupent une grande place dans l'étude quantitative des différentes caractéristiques du peuplement animal (Mathey et al., 1984). Le dispositif d'échantillonnage utilisé est représenté sur la Figure 3.

Trois transects de $200 \mathrm{~m}$ ont été délimités dans chaque type de culture. Vingt pièges de chaque type ont été disposés à équidistance $(10 \mathrm{~m})$ le long de chaque transect pour réaliser l'échantillonnage. Les pièges- 
fosses et les assiettes colorées situés au même point d'échantillonnage ont été placés parallèlement avec un écartement d'un mètre. $\mathrm{Au}$ total, 6 transects de $200 \mathrm{~m}$ ont été échantillonnés ( $\mathrm{n}=120$ échantillons de pièges fosses +120 assiettes colorées)

À l'aide d'une machette, des trous ont été creusés pour enterrer de façon verticale les pièges-fosses, dans lesquels tombaient les insectes fourrageurs. Par contre les assiettes colorées étaient placées sur le sol pour la capture des insectes volants.

Les pièges-fosses et les assiettes colorées ont été remplis au 2/3 tiers de leur volume avec de l'eau savonneuse empêchant les insectes de ressortir lorsqu'ils tombent dans les pièges.

$\mathrm{Au}$ bout de $48 \mathrm{~h}$ de piégeage, les insectes ont été collectés en faisant passer le contenu de chaque piège dans un petit tamis. Ils étaient stockés dans des piluliers contenant de l'alcool dilué à $70 \%$ qui portaient des étiquettes mentionnant la date, le type et le numéro du piège. Les échantillons d'insectes ont été ensuite transportés au laboratoire pour identification.

\section{Identification des insectes récoltés}

Les insectes collectés ont été identifiés à l'aide d'une loupe binoculaire de marque EISCO” aux grossissements X4. La

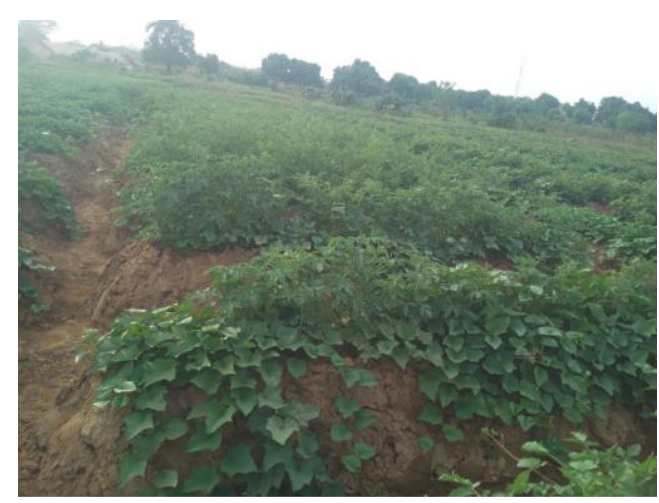

Figure 1: Parcelle de monoculture (patate douce). clé de détermination de Delvare et Aberlenc (1989) a été utilisée pour la séparation des échantillons en fonction des ordres.

\section{Analyses statistiques}

Le nombre d'ordre observé fait référence au nombre total d'ordres obtenu. Il a été calculé à partir du logiciel EXCEL. Aussi, les indices de diversité ont-elles été déterminés à partir du calcul des indices de diversité de Simpson (1-D) et de l'équitabilité (E) à l'aide du logiciel Past v. 2.17 (http://folk.uio.no/ohammer/past) .

La comparaison des moyennes de richesse et d'abondances d'insectes des deux systèmes culturaux a été effectuée en utilisant l'analyse de variance à un facteur (ANOVA 1) du logiciel Statistica 7.1' (www.statsoft.com).

L'indice de Jaccard a permis d'apprécier le niveau de similitudes entre la composition des deux peuplements d'insectes étudiés au moyen du logiciel EstimateS v.7.5 (http://purl.oclc.org/estimates). Anosim test a egalement été realisé pour tester la significativité de la variation de la structure des communautés des insectes. Le logiciel Past v. 2.17(http://folk.uio.no/ohammer/past) a permis de faire cette analyse.

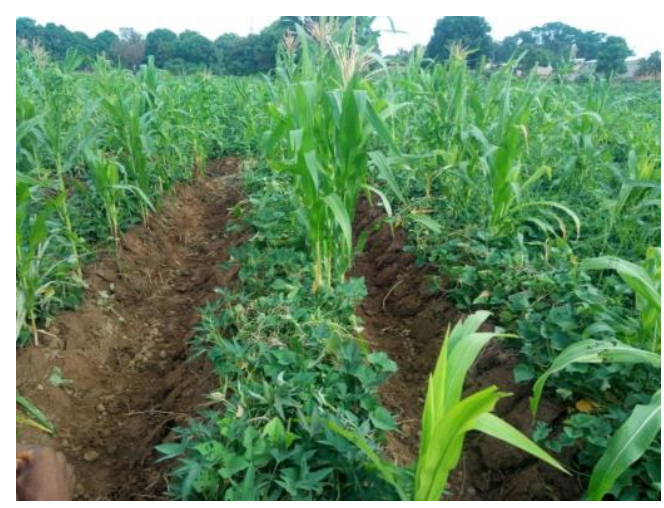

Figure 2: Parcelle de polyculture (patate douce et maïs). 

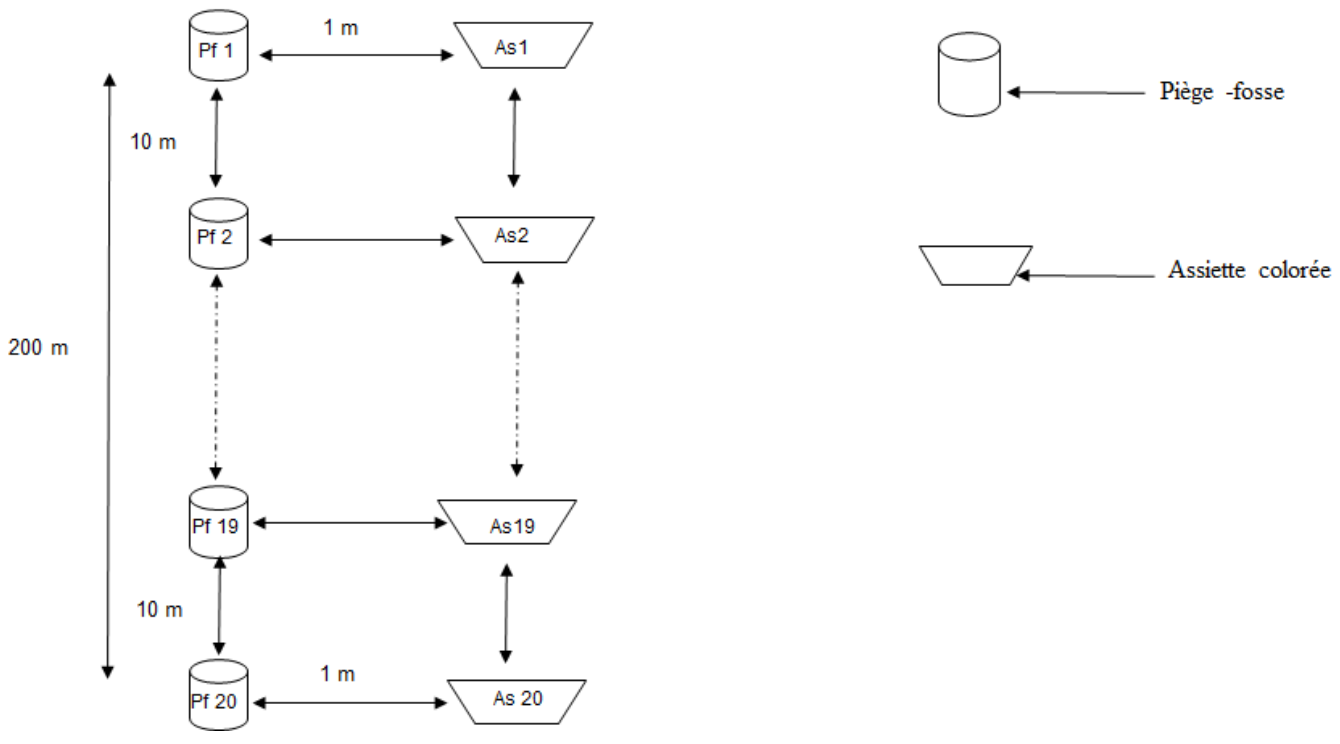

Figure 3 : Dispositif d'échantillonnage.

\section{RÉSULTATS}

\section{Richesse taxonomique et abondance des peuplements d'insectes dans les deux systèmes culturaux}

Au total, 9 ordres ont été récoltés dans les deux types d'habitats. Il s'agit des Hyménoptères, des Diptères, des Orthoptères, des Dictyoptères, des Coléoptères, des Hémiptères, des Plécoptères, des Lépidoptères et des Dermaptères (Tableau 1).

La monoculture renferme neuf (9) ordres d'insectes tandis que la polyculture n'en renferme que sept (7).

Au total 4837 individus, répartis en neuf ordres ont été collectés, avec 4112 individus en monoculture et 725 individus en polyculture (Tableau 2). L'analyse statistique révèle que cette différence entre les deux abondances est significative (test de Tukey : F $=7,61$ et $\mathrm{P}=0,007$ ) (Figure 4).

De manière détaillée, on note que seuls trois ordres d'insectes présentent des abondances significativement supérieures dans la monoculture par rapport à la polyculture. Ce sont les Hyménoptères (test de Tukey : $\mathrm{F}=7,13 ; \mathrm{P}<0,05)$, les Hémiptères (test de Tukey : $F=6,91 ; P=0,01$ ) et les Orthoptères (test de Tukey : $\mathrm{F}=4,33$; $\mathrm{P}$ $=0,04)$. Ces ordres sont plus abondants en monoculture qu'en polyculture (Tableau 1).

L'étude a révélé des valeurs des indices de diversité de Simpson et d'équitabilité élevées en polyculture contrairement aux monocultures où ces valeurs d'indices étaient relativement faibles (Tableau 2).

\section{Composition taxonomique des insectes dans les différentes pratiques culturales}

L'analyse de similitude entre les compositions taxonomiques des deux pratiques culturales montre une forte ressemblance avec un indice de Jaccard de 0,78 . ANOSIM test ne revèle aucune difference significative entre les deux pratiques culturales en terme de composition taxonomique $(R=0,09 ; \mathrm{P}=0,69)$. 


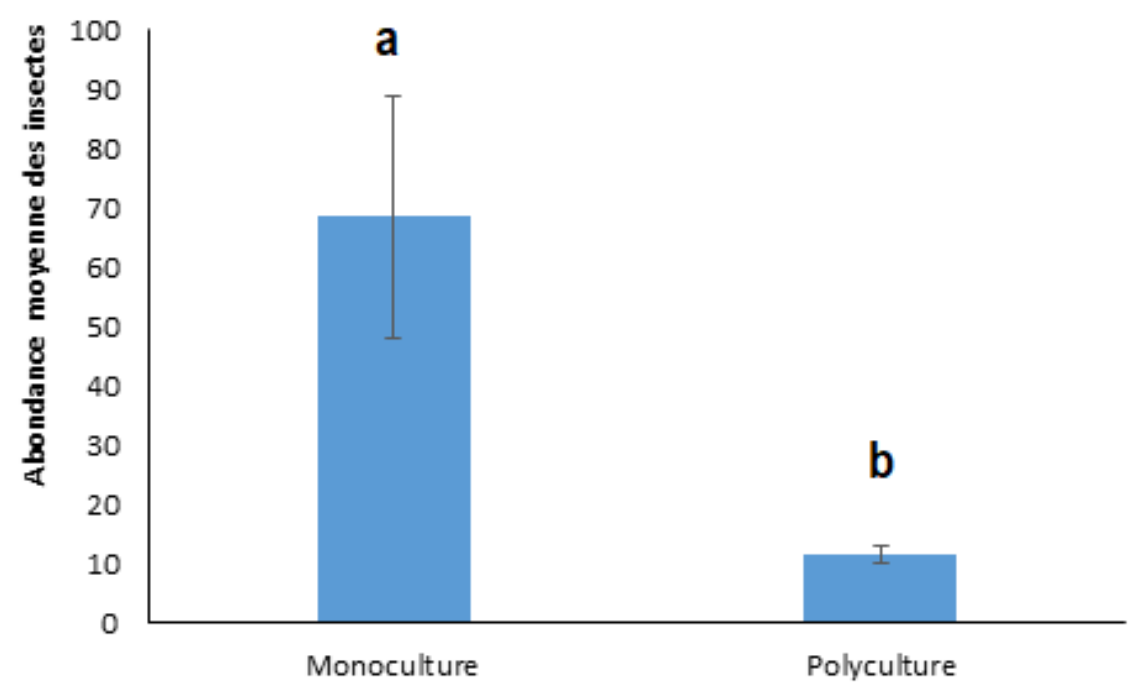

Figure 4 : Abondance moyennes des insectes en fonction des différents systèmes culturaux. Les histogrammes portant les lettres ( $\mathrm{a}$ et $\mathrm{b}$ ) diffèrent statistiquement pour $p=0,05$ (Test de Tukey).

Tableau 1 : Variation des abondances des groupes d'insectes récoltés dans les deux pratiques culturales.

\begin{tabular}{lcccc}
\hline Ordres & Monoculture & Polyculture & F & P \\
\hline Hyménoptères & 3535 & 315 & 7,13 & $\mathbf{0 , 0}$ \\
\hline Diptères & 312 & 270 & 0,32 & 0,57 \\
\hline Orthoptères & 55 & 28 & 4,33 & $\mathbf{0 , 0 4}$ \\
\hline Dictyoptères & 5 & 2 & 0,84 & 0,37 \\
\hline Coléoptères & 102 & 73 & 1,45 & 0,23 \\
\hline Hémiptères & 100 & 34 & 6,91 & $\mathbf{0 , 0 1}$ \\
\hline Plécoptères & 1 & 3 & 1,03 & 0,31 \\
\hline Lépidoptères & 1 & 0 & 1,00 & 0,32 \\
\hline Dermaptères & 1 & 0 & 1,00 & 0,32 \\
\hline
\end{tabular}

Tableau 2 : Richesse taxonomique, abondance et diversité des insectes dans les deux systèmes culturaux.

\begin{tabular}{lllll}
\hline & Richesse taxonomique & Abondance & $\begin{array}{l}\text { Indice de Simpson } \\
(\text { 1-D) }\end{array}$ & $\begin{array}{c}\text { Équitabilité } \\
\text { (E) }\end{array}$ \\
\hline Monoculture & 9 & 4112 & 0,25 & 0,20 \\
\hline Polyculture & 7 & 725 & 0,66 & 0,51 \\
\hline
\end{tabular}




\section{DISCUSSION}

L'étude menée a montré une diversité d'insectes dans les cultures vivrières dans la zone. Ce qui pourrait être dû au fait que cette zone présente des conditions agroécologiques favorables à la prolifération des insectes. Avec des valeurs d'indices de diversité de Simpson et d'équitabilité élevées en polycultures, il ressort que ce système cultural est relativement diversifié avec une bonne répartition des abondances au sein des différents ordres. Par contre, les monocultures sont peu diversifiées avec une mauvaise répartition des abondances.

Il a été constaté une forte abondance des insectes en monocultures contrairement aux polycultures. Cela peut s'expliquer par le fait que la transformation des habitats entraine l'extinction des espèces natives au profit des espèces ravageuses, vagabondes, invasives plus adaptées aux perturbations anthropiques, comme c'est le cas chez les fourmis (Graham et al., 2004 ; McKinney, 2008). Aussi, en monocultures on note moins d'ennemis naturels comparativement aux polycultures. Les travaux de Onzo (2016) ont montré que les associations culturales Jatropha-manioc et Jatropha-niebé ont un effet significatif sur la densité de population de la plupart des espèces d'insectes ravageurs de cette plante. Les associations culturales affecteraient l'usage des stimuli visuels par lesquels les insectes reconnaissent leurs plantes-hôtes. Aussi, elles entraineraient une perturbation des stimuli olfactifs (Tahvanainen et Root, 1972). Des études plus récentes effectuées par Francis et al. (2004) ont montré que l'(E)- $\beta$ - farnésène était le composé essentiel utilisé par un hyménoptère parasitoïde cherchant à localiser les œufs de son hôte, un hyménoptère Tenthredinidae. Cette information illustre la subtilité de l'information olfactive et permet d'imaginer les perturbations provoquées par l'association d'une plante non-hôte à une plante-hôte dans un champ sur les ravageurs.

Trois (3) ordres (Hyménoptères, Orthoptères et Hémiptères) étaient abondants en monoculture qu'en polyculture et cette abondance variait significativement. La plupart des Hyménoptères rencontrés au cours de cette étude était les fourmis, particulièrement les espèces Camponotus acvapimensis, Camponotus maculatus, pheidole megacephala, Monomorium pharaonis et Tetramorium sericeiventre. Elles étaient très abondantes en monoculture. Ces espèces sont pour la plupart opportunistes et vagabondes invasives. Elles ont une préférence pour les milieux perturbés ou les cultures mono spécifiques, traduisant ainsi leur forte abondance en monoculture (Koné et al., 2010).

Les Orthoptères étaient peu abondants en polycultures contrairement aux monocultures. Cela s'expliquerait par le fait que les polycultures constituent un habitat et un abri qui favorise et améliore la régulation naturelle par les auxiliaires. Aussi, faut-il noter que le microclimat créé par la polyculture n'est pas favorable au développement de la plupart des Orthoptères. Ces résultats vont dans le même sens de ceux de Graig et al. (1999) qui stipulent que ces insectes ont besoin des milieux ouverts et chauds pour leur reproduction et leur survie.

Les Hémiptères étaient faiblement représentés en polyculture. Cela pourrait s'expliquer par le fait qu'en polyculture, la présence des prédateurs impacterait sur leur abondance contrairement à la monoculture où beaucoup de leurs ennemis naturels ont été éliminés par l'utilisation de pesticide et aussi par l'homogénéisation de l'habitat qui leur est favorable

\section{Conclusion}

Les résultats de cette étude suggèrent que la polyculture est le système agricole qui permet de limiter la prolifération des insectes ravageurs. Toutefois, en raison des conditions des conditions climatiques variables dans le pays, il est souhaitable de conduire cette investigation dans d'autres régions pour confirmer ou infirmer les observations actuelles. Par ailleurs, l'identification des insectes au niveau spécifique peut faciliter la lutte contre les espèces ravageuses étant 
donné que les sensibilités sont variables d'une espèce à l'autre.

\section{CONFLIT D'INTÉRÊTS}

Nous déclarons qu'aucun conflit d'intérêts n'existe entre les auteurs. L'ordre des auteurs au niveau de l'article et le contenu du document a été validé à l'unanimité.

\section{CONTRIBUTIONS DES AUTEURS}

MK est l'investigateur principal et initiateur du projet ; YT et MLY ont participé à la rédaction du manuscrit; YAS a été en charge de collecter les données sur le terrain, de les saisir et les traiter ; ND et BK ont participé à la collecte des données sur le terrain.

\section{REMERCIEMENTS}

Nous remercions profondément les personnes et les organismes qui sont intervenus lors de cette étude. Il s'agit des paysans du village Gnalélékaha dans le département de Korhogo pour l'accueil et les facilités d'accès à leurs parcelles, et des étudiants en masters de Zoologie et développement Durable de UPGC. Nous exprimons nos sincères gratitudes à Dago Noel (Université Peleforo Gon Coulibaly, Côte d'Ivoire), pour son aide dans l'amélioration de la qualité de l'anglais du résumé.

\section{RÉFÉRENCES}

Anader (Agence nationale de développement Rural). 2009. La production vivrière : un enjeu national. Bulletin de liaison de l'agence nationale de développement rural. Bull. Liaison L'agence Natl. Dév. Rural 12p.

Auger J, Dugravot S, Naudin A, Abo-Ghalia A, Pierre D, Thibout E. 2002. Utilisation des composés allélochimiques des Allium en tant qu'insecticides. IOBC wprs Bull., 25: 295-306.

Onzo A, Seidou MS, Zoumarou-Wallis N, Datinon BD, Tamo M. 2016. Effets des associations culturales sur la dynamique de population des principaux insectes ravageurs et la production en graines de Jatropha curcas L. au Sud-Bénin. Int. J. Biol. Chem. Sci, 10(3) : 993-1006. DOI: http://dx.doi.org/10.4314/ijbcs.v10i3.7

Bianchi F, Booij CJH, Tscharntke T. 2006. Sustainable pest regulation in agricultural landscapes: a review on landscape composition, biodiversity and natural pest control. Proceedings of Royal Society B: Biological Sciences, 273(1595): 1715-1727. DOI: https:// http://dx.doi.org/10.1098/rspb.2006.3530 Chaplin-Kramer R, O’Rourke ME, Blitzer EJ, Kremen C. 2011. A meta-analysis of crop pest and natural enemy response to landscape complexity. Ecological Letters, 14: 922-932. DOI: http://dx.doi.org/10.1111/j.14610248.2011.01642.x

Chaplin-Kramer R, de Valpine P, Mills NJ, Kremen C. 2013. Detecting pest control services across spatial and temporal scales. Agriculture, Ecosystems and Environment, 181: 206-212. DOI: http://dx.doi.org/10.1016/j.agee.2013.10. 007

Choudourou DC, Agbaka A, Adjakpa JB, Koutchika RE, Adjalian EJN. 2012. Inventaire préliminaire de l'entomofaune des champs de tomates (Lycopersicon esculentum Mill) dans la Commune de Djakotomey au Bénin. Int. J. Biol. Chem. Sci, 6(4): 1798-1804. DOI: http://dx.doi.org/10.4314/ijbcs.v6i4.34

Delvare G, Aberlenc HP. 1989. Les insectes d'Afrique et d'Amérique tropicale. Clé pour la reconnaissance des familles. Monpelier, France: Ed CIRAD. 298 p.

Diatte M, Brévault T, Sall-Sy D, Diarra K. 2016. Des pratiques culturales influent sur les attaques de deux ravageurs de la tomate dans les Niayes au Sénégal. Int. J. Biol. Chem. Sci., 10(2): 681-693. DOI: http://dx.doi.org/10.4314/ijbcs.v10i2.19

FAO. 2015. Cadre de programmation pays 2012-2015, Côte d'Ivoire. FAO doc. 29p. http://www.fao.org/3/a-be840f.pdf. 
Francis F, Lognay G, Haubruge E. 2004. Olfactory responses to aphid and host plant volatile releases: (E)- $\beta$-Farnesene an effective kairomone for the predator Adalia bipunctata. Journal of Chemical Ecology, 30(4): 741-755. DOI: http://dx.doi.org/10.1023/B:JOEC.00000 28429.13413.a2.

Graham JH, Hughie HH, Jones S, Wrinn K, Krzysik AJ, Duda JJ, Balbach H. 2004. Habitat disturbance and the diversity and abundance of ants (Formicidae) in the Southeastern FallLine Sandhills. Journal of Insect Science, 4: 30. https://www.ncbi.nlm.nih.gov/pmc/articl es/PMC1081558/

Craig DP, Bock CE, Bennett BC, Bock JH. 1999. Habitat relationships among grasshoppers (Orthoptera: Acrididae) at the western limit of the Great Plains in Colorado. American Midland Naturalist, 142(2): 314-327. DOI: http://dx.doi.org/10.1674/00030031(1999)142[0314:HRAGOA]2.0.CO; 2

Hautier L, Patiny S, Thomas-Odjo A, Gaspar C. 2003. Evaluation de la biodiversité de l'entomofaune circulante au sein d'associations culturales au Nord Bénin, Notes fauniques de Gembloux, $\mathrm{n}^{\circ} 52$ : 39-51

Kone M, Konate S, Yeo K, Kouassi KP, Linsenmair KE. 2010. Diversity and abundance of terrestrial ants along a gradient of land use intensification in a transitional forest-savannah zone of Côte d'Ivoire. Journal of Applied Biosciences, 29: 1809-1827.

DOI : http://www.m.elewa.org/JABS/2010/29/ 7.pdf

Mailafiya DM, Degr MM, Maina YT, Gadzama UN, Galadima IB. 2014. Preliminary Studies on Insect Pest Incidence on Tomato in Bama, Borno State, Nigeria. Int. Let. Nat. Scie., 5: 4554. DOI : http://dx.doi.org/ 10.18052/www.scipress.com/ILNS.10.45 Mathey W, Dellasanta E, Wannenmacher C. 1984. Manuel Pratique d'Ecologie. Payot: Lausanne, Suisse. 204 p.

McKinney ML. 2008. Effects of urbanization on species richness: A review of plants and animals. Urban Ecosystems, 2: 161176.

DOI: http://dx.doi.org/10.1007/s11252-0070045-4

Tahvanainen JO, Root RB. 1972. The influence of vegetational diversity on the population ecology of a specialized herbivore, Phyllotreta cruciferae (Coleoptera:Chrysomelidae). Oecologia, 10: 321-346.

Umeh VC, Kuku FO, Nwanguma EI, Adebayo OS, Manga AA. 2002. A survey of the insect pests and farmers' practices in the cropping of tomato in Nigeria. Tropicultura, 20(4): 181-186. 